\title{
Amostragens Longitudinais Alternativas para a Determinação da Densidade Básica em Clones de Eucalyptus sp.
}

\author{
Allan Motta Couto, Thiago de Paula Protásio, \\ Aliny Aparecida dos Reis, Paulo Fernando Trugilho \\ Departamento de Ciências Florestais - DCF, Universidade Federal de Lavras - UFLA
}

\begin{abstract}
RESUMO
Os objetivos desse trabalho foram: a) determinar o padrão de variação longitudinal da densidade básica; b) avaliar os principais métodos de amostragem longitudinal; e c) propor novas alternativas amostrais longitudinais representativas da densidade básica média. Foram retirados discos da base, $1,30 \mathrm{~m}$ (DAP) e, a partir deste ponto, de metro em metro até a altura comercial. Foram ajustados modelos lineares explicativos da variação longitudinal da densidade básica. O modelo polinomial de $5^{\circ}$ ordem foi o que melhor explicou o padrão de variação longitudinal da densidade básica. Esse modelo foi utilizado na predição da densidade básica nas alturas relativas de 1 a $100 \%$ da altura comercial. A posição de $79 \%$ da altura comercial foi a mais precisa dentre as avaliadas. As alternativas amostrais A (5, 25, 27, 78 e 79\%), B $(5,26,27,78$ e $79 \%)$ e $C(5,26,27,78$ e $80 \%)$ foram mais adequadas às amostragens comumente utilizadas.
\end{abstract}

Palavras-chave: eucalipto, modelo polinomial, variação.

\section{Longitudinal Alternative Sampling for Determination of the Basic Density in Eucalyptus sp. Clones}

\begin{abstract}
The purposes of this research were: a) to determine the longitudinal variation standard of basic density; b) to evaluate the main longitudinal sampling methods and; c) to propose new alternatives of representative longitudinal sampling of the average basic density. Disks were obtained from the tree basis, in the $1.30 \mathrm{~m}(\mathrm{DBH})$ position, and at every meter from this point until commercial height. Explanatory linear models of basic density longitudinal variation were adjusted. The polynomial model of fifth order was the one that better explained the longitudinal variation of basic density. This model was used for the prediction of basic density at relative heights from 1 to $100 \%$ of the commercial height. The position at $79 \%$ of the commercial height was the most precise among the ones evaluated. The sampling alternatives A $(5,25,27,78$ and $79 \%), B(5,26,27,78$ and $79 \%)$ and C $(5,26,27,78$ and $80 \%)$ were more adequate to the samplings commonly used.
\end{abstract}

Keywords: eucalypt, polynomial model, variation. 


\section{INTRODUÇÃO}

A madeira, por ser um material muito variável, permite ser destinada para as mais diversas finalidades. Cada finalidade atribuída à madeira exige características específicas dessa matéria-prima, que contribuem para a seleção de espécies mais adequadas para determinado uso, com o processo produtivo e a qualidade do produto final. Diante disso, índices referentes à qualidade da madeira têm sido cada vez mais apreciados com o objetivo de melhor caracterizar o material madeira.

A densidade básica (DB) é uma propriedade física da madeira de fácil determinação e, por se correlacionar com as demais propriedades, tem sido considerada como o principal índice de qualidade deste material. Esta propriedade apresenta alta influência sobre os mais diversos processos produtivos dos quais a madeira participa.

A variação das propriedades da madeira ocorre sob diversos níveis. Ocorre entre espécies, entre indivíduos de mesma espécie, entre indivíduos de mesmo clone e até dentro do mesmo fuste. A variação radial e longitudinal das propriedades da madeira são as principais fontes de variação dentro de um fuste.

Quanto à densidade básica, é possível encontrar vários padrões de variação dessa propriedade na literatura para pinus ou eucalipto, tanto base-topo quanto medula-casca (Mattos et al., 2011; Couto et al., 2010; Protásio et al., 2010; Trugilho et al., 2010, 2008; Arango Alzate et al., 2005; Lima et al., 1992).

Diante da grande variação dessa propriedade, não há consenso na literatura sobre as melhores posições de amostragem para a determinação da densidade básica média da árvore. Contudo, vários pesquisadores adotam a amostragem em cinco posições longitudinais, ou seja, a 0, 25, 50, 75 e 100\% da altura comercial ou total (Mattos et al., 2011; Trugilho et al., 2010; Arango Alzate et al., 2005; Oliveira et al., 2005). A amostragem de metro em metro também tem sido utilizada para a determinação da densidade básica média da árvore (Trugilho et al., 2010; Franco et al., 1998).

Segundo Trugilho et al. (2010), o método tradicional $(0,25,50,75$ e $100 \%$ da altura) apresentou tendência de subestimar a densidade básica com relação à amostragem de metro em metro, principalmente nos indivíduos pertencentes às maiores classes de diâmetro para um clone híbrido de Eucalyptus.

Já a amostragem proposta por Downes et al. (1997), considerada uma amostragem alternativa para a determinação da densidade básica média, consiste na retirada de discos a 2, 10, 30, 50 e 70\% em relação à altura total das árvores e tem sido utilizada em materiais pertencentes ao gênero Eucalyptus (Neves et al., 2011; Protásio et al., 2010, 2011; Trugilho et al., 2010).

Dessa forma, uma amostragem adequada e que melhor represente a densidade básica média da árvore é de grande importância. Uma amostragem inadequada pode levar a inferências incorretas sobre a qualidade da madeira, que se extrapolada para uma população, pode gerar inconvenientes econômicos. Sendo a variação base-topo uma das principais dentro de um fuste, a amostragem longitudinal se torna muito importante para determinação da densidade básica média de um indivíduo.

Diante do exposto, o objetivo deste trabalho foi determinar o padrão de variação longitudinal da densidade básica de clones de Eucalyptus, avaliar os principais métodos amostrais utilizados na determinação da densidade básica média da árvore e propor alternativas amostrais longitudinais que melhor representem essa propriedade.

\section{MATERIAL E MÉTODOS}

\subsection{Material biológico}

Foram utilizados 46 clones de Eucalyptus sp. aos 42 meses de idade provenientes de teste clonal pertencente à empresa ArcelorMittal Bioenergia Ltda., localizada em Martinho Campos, região centro-oeste de Minas Gerais, Brasil. Utilizaram-se cinco árvores por clone, totalizando 230 árvores.

\subsection{Amostragem longitudinal}

As árvores foram abatidas e destas foram retirados discos de $\pm 2,5 \mathrm{~cm}$ de espessura na base, a 1,30 m (DAP) e, a partir desse ponto, de metro em metro até a altura comercial $(\mathrm{Hc})$, sendo esta considerada com diâmetro de $5 \mathrm{~cm}$ com casca. 


\subsection{Determinação da densidade básica}

Os discos foram seccionados em quatro cunhas passando pela medula, sendo utilizadas duas opostas para a determinação da densidade básica (DB), conforme descrito pela norma NBR 11941 (ABNT, 2003). A densidade básica média (DBmed) da árvore foi considerada como sendo a média aritmética de todas as posições longitudinais de amostragem.

\subsection{Determinação do padrão de variação longitudinal da densidade básica}

Foram avaliados quatro modelos lineares para caracterizar o padrão de variação longitudinal da densidade básica para cada árvore amostrada, o modelo quadrático (Equação 1), o cúbico (Equação 2) e os modelos polinomiais de $4^{\circ}$ e $5^{\circ}$ ordem (Equações 3 e 4, respectivamente).

$$
\begin{aligned}
& \mathrm{DB}=\beta_{0}+\beta_{1}(\mathrm{~h})^{1}+\beta_{2}(\mathrm{~h})^{2}+\varepsilon \\
& \mathrm{DB}=\beta_{0}+\beta_{1}(\mathrm{~h})^{1}+\beta_{2}(\mathrm{~h})^{2}+\beta 3(\mathrm{~h})^{3}+\varepsilon \\
& \mathrm{DB}=\beta_{0}+\beta_{1}(h)^{1}+\beta_{2}(h)^{2}+\beta_{3}(h)^{3}+\beta_{4}(h)^{4}+\varepsilon \\
& D B=\beta_{0}+\beta_{1}(h)^{1}+\beta_{2}(h)^{2}+\beta_{3}(h)^{3}+\beta_{4}(h)^{4}+\beta_{5}(h)^{5}+\varepsilon
\end{aligned}
$$

em que: $D B$ é a densidade básica $\left(\mathrm{g} / \mathrm{cm}^{3}\right)$ na altura relativa de interesse; $\beta_{0}, \beta_{1}, \beta_{2}, \beta_{3}, \beta_{4}$ e $\beta_{5}$ são os coeficientes dos modelos; h é a posição longitudinal da árvore $(\mathrm{m})$; $\varepsilon$ é o erro do modelo.

O modelo que melhor caracterizou o padrão de variação longitudinal da DB foi utilizado na predição da DB em função das alturas relativas que variaram de 1 a $100 \%$ da altura total do fuste $(\mathrm{Hc})$.

A densidade estimada nos pontos ao longo do fuste foi comparada à densidade básica média individual observada, dando origem, assim, aos desvios referentes a cada altura relativa para todos os indivíduos. Assim pôde-se selecionar as alturas relativas que melhor representaram a DBmed. O somatório residual próximo de zero, juntamente com a baixa magnitude do desvio padrão, é um indício de alta precisão na inferência sobre a DBmed, baseado em apenas um ponto longitudinal.

\subsection{Amostragens longitudinais alternativas}

Foram selecionadas sete alturas relativas que melhor representam a DBmed individual em função do desvio padrão e somatório dos desvios. Com as alturas selecionadas, foram feitas combinações entre elas, visando à determinação de alternativas amostrais baseadas em cinco posições longitudinais, assim sendo, foram propostos 21 métodos de amostragem longitudinal no fuste das árvores.

Para fins comparativos, as amostragens pontuais tiveram como parâmetro a amostragem realizada apenas a $1,30 \mathrm{~m}$ do solo (DAP). Já as amostragens baseadas em cinco pontos longitudinais foram comparadas à amostragem convencional $(0,25,50,75$ e $100 \%$ de Hc) e à amostragem proposta por Downes et al. (1997) (2, 10, 30, 50 e 70\% de Hc).

\subsection{Análise estatística}

A avaliação da precisão de cada alternativa amostral foi baseada nos testes dos desvios, para tal, utilizou-se o software estatístico $\mathrm{R}$ 2.9.2 (R Developement Core Team, 2009). Dentre os critérios de seleção da alternativa amostral, utilizouse o desvio padrão e soma dos desvios e adequação aos pressupostos residuais de homogeneidade de variância (homocedasticidade) e comportamento normal (normalidade).

\section{RESULTADOS E DISCUSSÃO}

\subsection{Padrão de variação longitudinal da densidade básica}

A Figura 1 apresenta as variações longitudinais da densidade básica obtidas pelos modelos ajustados, sendo os modelos quadrático, cúbico, polinomial de $4^{\circ}$ e $5^{\circ}$ ordem representados por a, b, c e d, respectivamente.

Pode-se observar que o comportamento dos modelos B, C e D são semelhantes, porém, em análise residual, o modelo polinomial de $5^{\circ}$ ordem apresenta uma suave superioridade na predição da DB em função da altura, quando comparado aos demais modelos. $\mathrm{O}$ padrão de variação longitudinal da DB caracterizado pelo modelo polinomial de 5 foi observado na grande maioria dos indivíduos avaliados. 
Arango Alzate et al. (2005), ao estudar o padrão de variação longitudinal da densidade básica de clones de Eucalyptus grandis, observaram um decréscimo da densidade básica até $50 \%$ da altura comercial seguida de um aumento até o ápice da tora. Já Oliveira et al. (2005) observaram em árvores de Eucalyptus grandis um decréscimo da densidade básica da madeira da base até um quarto da altura comercial seguida de um aumento até o topo da árvore.

Os padrões de variação descritos por esses autores diferem dos encontrados neste trabalho, devido possivelmente aos diferentes clones utilizados, locais de cultivo e tratamentos silviculturais adotados.

\subsection{Amostragem longitudinal pontual}

As alturas relativas que melhor representaram a DBmed foram a 5, 25, 26, 27, 78, 79 e $80 \%$ da altura comercial. A Tabela 1 apresenta a soma, desvios padrão, valores de máximo e mínimo dos desvios a $1,30 \mathrm{~m}$ do solo (DAP) e nas alturas que melhor representaram a DBmed. Apresenta também o resultado dos testes para avaliação da presença de normalidade e homocedasticidade dos desvios.

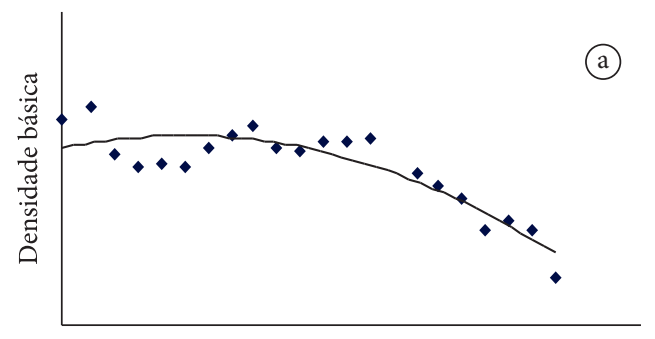

Altura

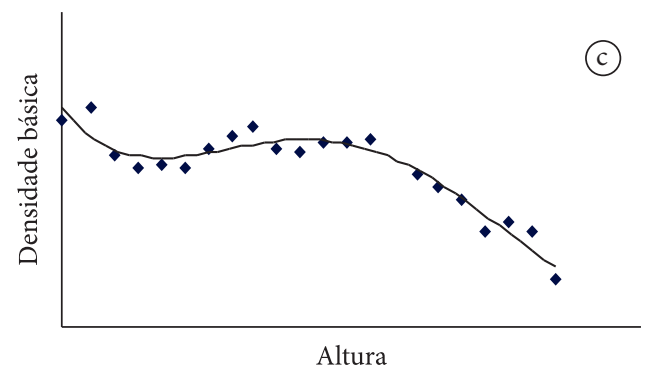

Observa-se na Tabela 1 que apenas as alturas relativas a 78,79 e $80 \%$ da altura comercial $(\mathrm{Hc})$ atenderam aos dois pressupostos dos desvios simultaneamente. O DAP, por sua vez, além de não atender a nenhum dos pressupostos, apresentou o segundo maior desvio padrão e somatório dos desvios mais distante de zero, inferindo assim que o DAP pode não ser o melhor ponto de amostragem para representar DBmed. A altura relativa a $79 \%$ de Hc apresentou o terceiro somatório dos desvios mais próximo a zero, que, aliado à adequação aos pressupostos, indica que esta é a que melhor representa a DBmed.

As Figuras 2 e 3 apresentam a relação entre a DBmed e DB no ponto considerado (a) e a distribuição gráfica dos desvios (b) a 79\% de Hc e no DAP, respectivamente.

Pode-se observar que o comportamento dos dados assemelha-se ao da reta ideal (em preto), tal resultado sugere que a DB na posição $79 \%$ de Hc está próxima à densidade básica média das árvores. Nota-se, observando o gráfico de distribuição residual, a proximidade dos desvios $\left(\mathrm{g} / \mathrm{cm}^{3}\right)$ com o eixo X (DBmed); este é um indício da precisão da estimativa da DBmed utilizando apenas um único ponto amostral.
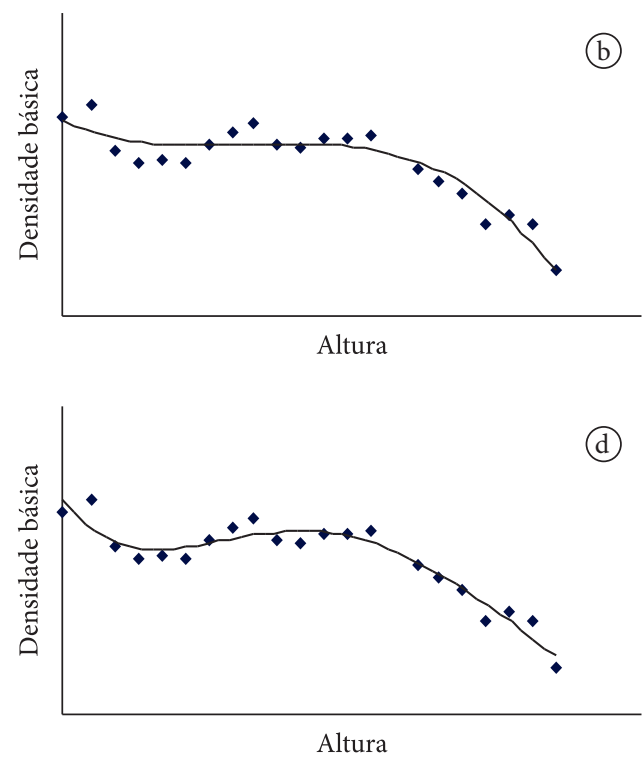

Figura 1. Padrões de variação longitudinal da DB caracterizados pelos modelos quadrático (a), cúbico (b), polinomial de $4^{\circ}(\mathrm{c})$ e $5^{\circ}(\mathrm{d})$ ordem ajustados.

Figure 1. Longitudinal variation patterns of BD characterized by adjusted quadratic (a), cubic (b), polynomial of $4^{\circ}$ (c) and $5^{\circ}$ order models. 

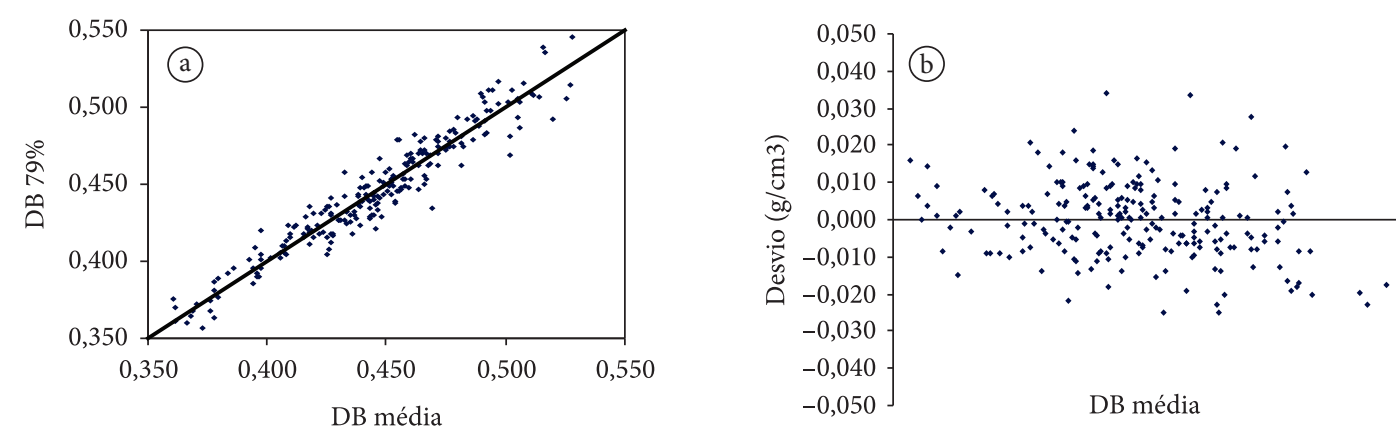

Figura 2. Relação entre os dados de DB med e DB a 79\% de Hc (a) e distribuição gráfica dos desvios (b).

Figure 2. Relation between data of average BD and BD at 79\% (a) and graphic distribution of the deviations (b).
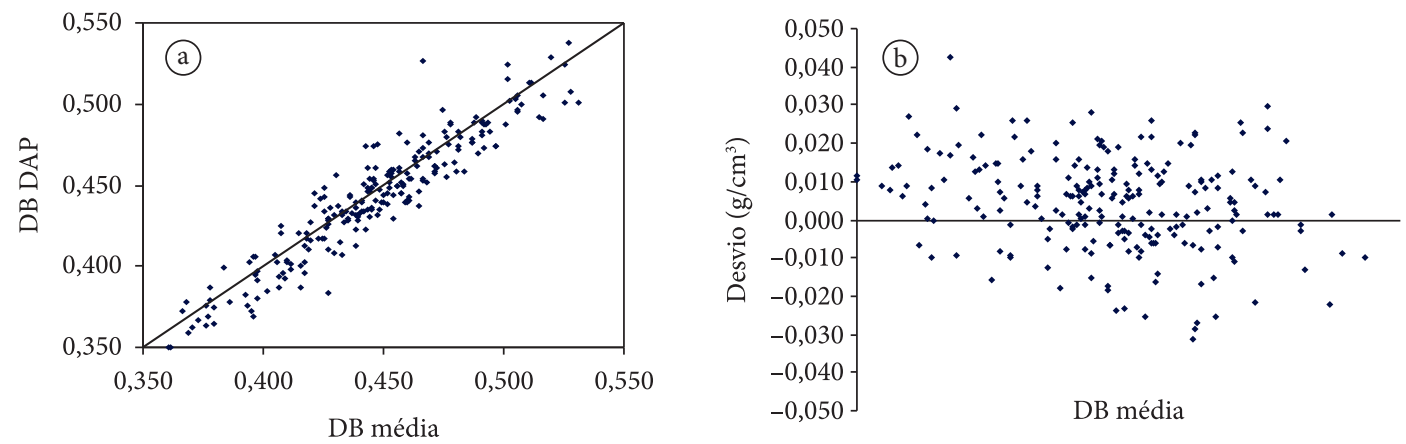

Figura 3. Relação entre os dados de DB med e DB no DAP (a) e distribuição gráfica dos desvios (b).

Figure 3. Relation between data of average $\mathrm{BD}$ and $\mathrm{BD}$ at $\mathrm{DBH}$ (a) and graphic distribution of the deviations (b).

Tabela 1. Estatísticas utilizadas para avaliar a estimativa da densidade básica média por meio da amostragem de apenas uma posição na árvore.

Table 1. Statistics used to evaluate the estimation of average basic density by sampling only one position in the tree.

\begin{tabular}{ccccccc}
\multirow{2}{*}{$\begin{array}{c}\text { Amostragem } \\
\text { pontual }\end{array}$} & \multicolumn{2}{c}{ Desvios } & \multicolumn{2}{c}{$\begin{array}{c}\text { Testes de pressuposição } \\
\text { residual }\end{array}$} \\
\cline { 2 - 7 } & $\begin{array}{c}\text { Soma } \\
\left(\mathbf{g} / \mathbf{c m}^{3}\right)\end{array}$ & $\begin{array}{c}\mathbf{S}_{\mathbf{x y}} \\
\left(\mathbf{g} / \mathbf{c m}^{3}\right)\end{array}$ & $\begin{array}{c}\text { Máximo } \\
\left(\mathbf{g} / \mathbf{c m}^{3}\right)\end{array}$ & $\begin{array}{c}\text { Mínimo } \\
\left(\mathbf{g} / \mathbf{c m}^{3}\right)\end{array}$ & W & GQ \\
\hline $5 \%$ & $-0,031$ & 0,014 & 0,051 & $-0,072$ & $0,9664^{*}$ & $1,5701^{*}$ \\
\hline $25 \%$ & 0,501 & 0,010 & 0,039 & $-0,028$ & $0,9897^{\text {ns }}$ & $2,0550^{*}$ \\
\hline $26 \%$ & 0,227 & 0,010 & 0,037 & $-0,029$ & $0,9902^{\text {ns }}$ & $1,7901^{*}$ \\
$27 \%$ & $-0,047$ & 0,009 & 0,035 & $-0,031$ & $0,9899^{\text {ns }}$ & $1,8827^{*}$ \\
\hline $78 \%$ & $-0,227$ & 0,010 & 0,031 & $-0,030$ & $0,9931^{\text {ns }}$ & $0,8107^{\text {ns }}$ \\
\hline $79 \%$ & $-0,049$ & 0,011 & 0,034 & $-0,030$ & $0,9931^{\text {ns }}$ & $0,7963^{\text {ns }}$ \\
\hline $80 \%$ & 0,138 & 0,011 & 0,037 & $-0,031$ & $0,9931^{\text {ns }}$ & $0,7692^{\text {ns }}$ \\
\hline DAP & 0,918 & 0,013 & 0,043 & $-0,060$ & $0,9711^{*}$ & $1,6057^{*}$ \\
\hline
\end{tabular}

$\mathrm{S}_{\mathrm{xy}}$ : desvio padrão; DAP: densidade básica a $1,30 \mathrm{~m}$ do solo; $\mathrm{W}^{\star} \mathrm{e} \mathrm{GQ}^{*}$ : ausência de normalidade e homocedasticidade dos desvios, respectivamente.

\subsection{Amostragem utilizando à média aritmética de cinco pontos longitudinais}

As alternativas amostrais avaliadas tiveram origem nas combinações possíveis de cinco pontos utilizando os sete que melhor representaram a DBmed. A Tabela 2 apresenta as alternativas amostrais avaliadas.

Apesar da proximidade entre a DBmed e a DB a $79 \%$ da Hc, a inferência sobre a DBmed com base apenas em um único ponto é menos confiável 
se comparada à estimativa da densidade básica considerando-se vários pontos de amostragem. Dessa forma, a utilização de mais pontos amostrais, além de possibilitar a redução dos desvios, aumenta a confiabilidade do resultado.

A Tabela 3 apresenta a soma, desvios padrão, valores de máximo e mínimo dos desvios das alternativas amostrais, que, em teoria, melhor representaram a DBmed. Apresenta também o resultado dos testes Shapiro-Wilk (W) e GoldfeldQuandt (GQ) para avaliação da presença de normalidade e homocedasticidade dos desvios, respectivamente.

Observa-se na Tabela 3 que a amostragem convencional e a proposta por Downes et al. (1997) não atenderam aos pressupostos dos desvios e apresentaram seu somatório mais distante de zero. Tais resultados inferem que estes pontos de amostragem em conjunto podem não representar com precisão a DBmed dos clones de eucalipto avaliados.

Em contrapartida, as amostragens alternativas relativas aos números 7,11 e 12 atenderam aos pressupostos dos desvios e apresentaram o desvio padrão e somatório dos desvios próximo de zero, logo, podem ser alternativas amostrais mais adequadas para representar a DBmed das árvores avaliadas.

As Figuras 4-8 apresentam a relação entre os dados de DBmed e DB determinada pelas alternativas amostrais consideradas e a distribuição gráfica dos desvios das amostragens 7, 11, 12, a proposta por Downes et al. (1997) e a convencional, respectivamente.

Tabela 2. Alternativas amostrais estabelecidas pelas combinações possíveis das alturas relativas que melhor representaram a DBmed.

Table 2. Sampling alternatives established by possible combinations of relative height that better represent average BD.

\begin{tabular}{cccc}
$\begin{array}{c}\text { Alternativa } \\
\text { amostral }\end{array}$ & $\begin{array}{c}\text { Alturas relativas } \\
\text { utilizadas (\%) }\end{array}$ & $\begin{array}{c}\text { Alternativa } \\
\text { amostral }\end{array}$ & $\begin{array}{c}\text { Alturas relativas } \\
\text { utilizadas }(\%)\end{array}$ \\
\hline 1 & $5 / 25 / 26 / 27 / 78$ & 12 & $5 / 26 / 27 / 78 / 80$ \\
2 & $5 / 25 / 26 / 27 / 79$ & 13 & $5 / 26 / 27 / 79 / 80$ \\
3 & $5 / 25 / 26 / 27 / 80$ & 14 & $5 / 26 / 78 / 79 / 80$ \\
4 & $5 / 25 / 26 / 78 / 79$ & 15 & $5 / 27 / 78 / 79 / 80$ \\
\hline 5 & $5 / 25 / 26 / 78 / 80$ & 16 & $25 / 26 / 27 / 78 / 79$ \\
6 & $5 / 25 / 26 / 79 / 80$ & 17 & $25 / 26 / 27 / 78 / 80$ \\
\hline 7 & $5 / 25 / 27 / 78 / 79$ & 18 & $25 / 26 / 27 / 79 / 80$ \\
\hline 8 & $5 / 25 / 27 / 78 / 80$ & 19 & $25 / 26 / 78 / 79 / 80$ \\
\hline 9 & $5 / 25 / 27 / 79 / 80$ & 20 & $25 / 27 / 78 / 79 / 80$ \\
\hline 10 & $5 / 25 / 78 / 79 / 80$ & 21 & $26 / 27 / 78 / 79 / 80$ \\
\hline 11 & $5 / 26 / 27 / 78 / 79$ & - & - \\
\hline
\end{tabular}
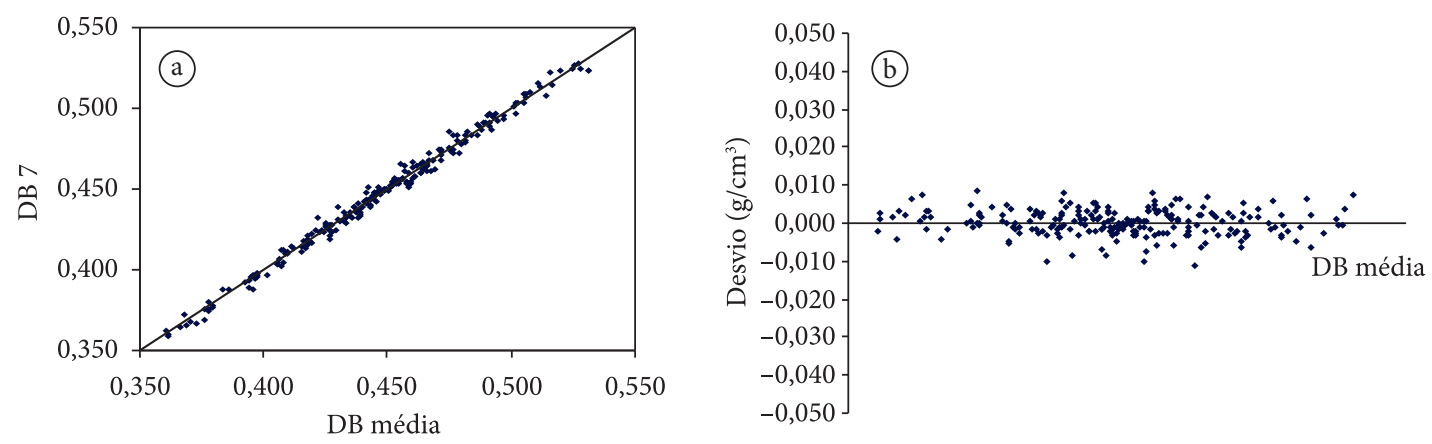

Figura 4. Relação entre os dados de DBmed e DB determinada pela alternativa amostral 7 (a) e distribuição gráfica dos desvios (b).

Figure 4. Relation between data of average BD and BD determined by the sampling alternative 7 (a) and graphic distribution of the deviations (b). 
Analisando o primeiro gráfico de cada figura, pode-se observar que o comportamento dos dados assemelha-se ao da reta ideal (em preto), tal resultado sugere que a $\mathrm{DB}$ nos pontos avaliados está próxima a DBmed. Nota-se, observando o gráfico de distribuição residual, a proximidade dos desvios $\left(\mathrm{g} / \mathrm{cm}^{3}\right)$ com o eixo X (DBmed), este é um indício da alta precisão dos modelos na estimativa da DBmed, utilizando cinco pontos longitudinais de amostragem.

Observando os gráficos de dispersão dos desvios referentes à amostragem proposta por Downes et al. (1997) e à convencional (Figura 7 e 8), nota-se uma tendência de superestimação e subestimação da DBmed, respectivamente. Tal comportamento não é observado para as alternativas amostrais 7, 11 e 12 .
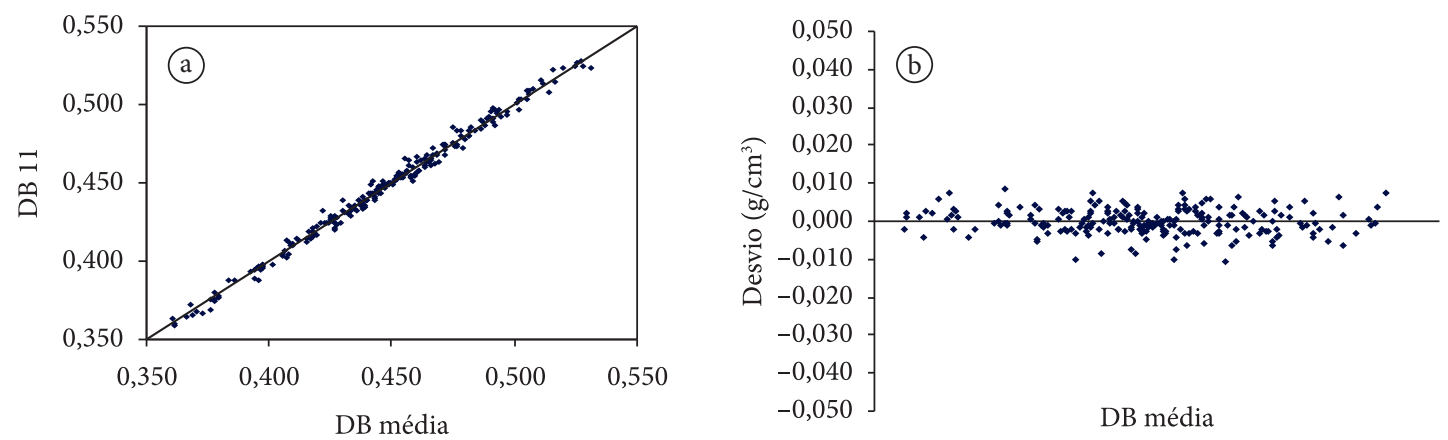

Figura 5. Relação entre os dados de DBmed e DB determinada pela alternativa amostral 11 (a) e distribuição gráfica dos desvios (b).

Figure 5. Relation between data of average BD and BD determined by sampling alternative 11 (a) and graphic distribution of the deviations (b).
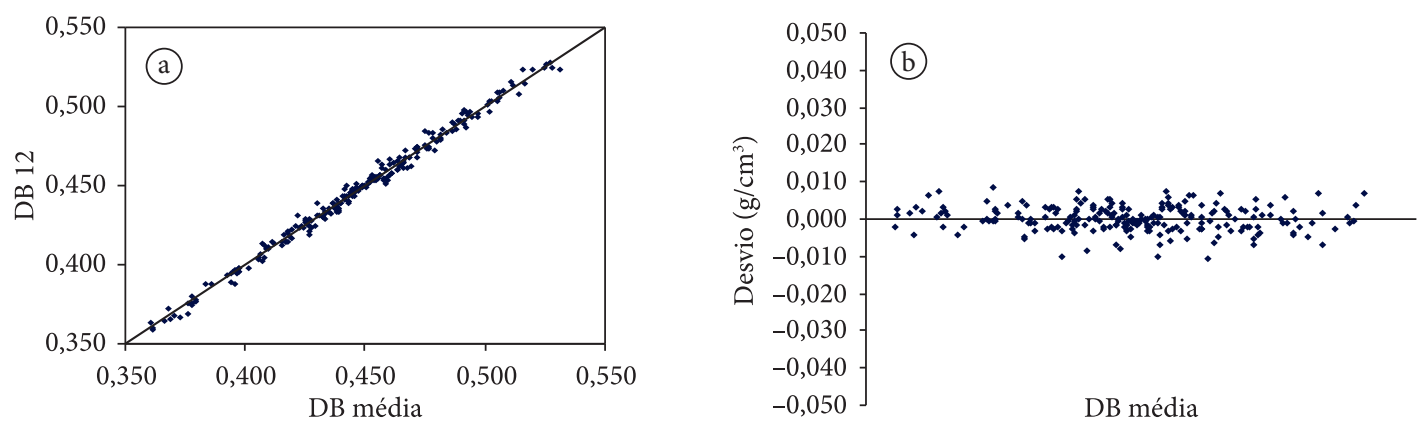

Figura 6. Relação entre os dados de DBmed e DB determinada pela alternativa amostral 12 (a) e distribuição gráfica dos desvios (b).

Figure 6. Relation between data of average BD and BD determined by sampling alternative 12 (a) and graphic distribution of the deviations (b).
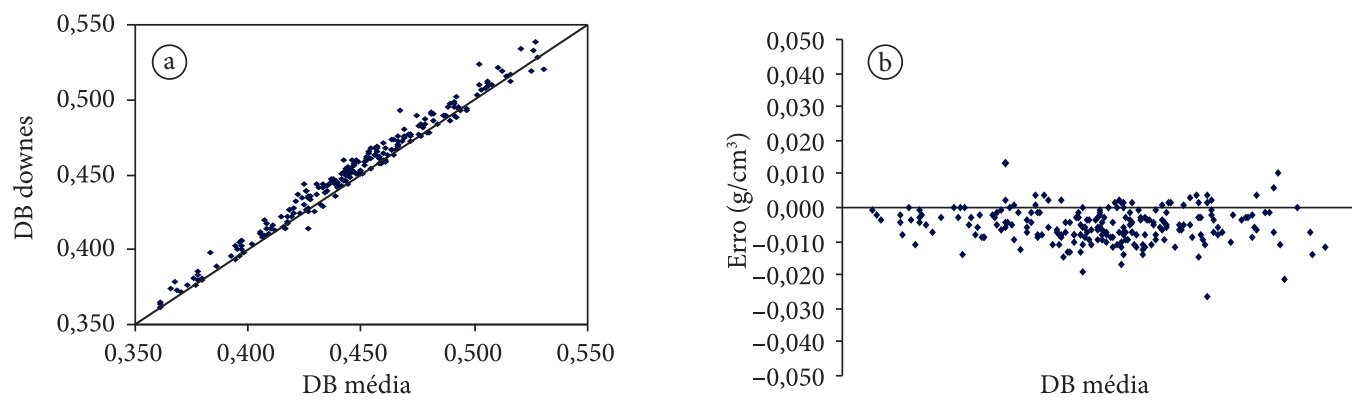

Figura 7. Relação entre os dados de DBmed e DB determinada pela amostragem proposta por Downes et al. (1997) (a) e distribuição gráfica dos desvios (b).

Figure 7. Relation between data of average BD and BD determined by the sampling proposed by Downes et al. (1997) (a) and graphic distribution of the deviations (b). 
Tabela 3. Estatísticas utilizadas para avaliar a estimativa da densidade básica média por meio das alternativas amostrais propostas.

Table 3. Statistics used for evaluation the estimation of average basic density by the proposed sampling alternatives.

\begin{tabular}{|c|c|c|c|c|c|c|}
\hline \multirow[b]{2}{*}{ Amostragem } & \multicolumn{4}{|c|}{ Desvios } & \multicolumn{2}{|c|}{ Testes dos desvios } \\
\hline & $\begin{array}{l}\text { Soma } \\
\left(\mathrm{g} / \mathrm{cm}^{3}\right)\end{array}$ & $\begin{array}{c}S_{x y} \\
(\mathrm{~g} / \mathrm{cm} 3)\end{array}$ & $\begin{array}{c}\text { Máximo } \\
\left(\mathrm{g} / \mathrm{cm}^{3}\right)\end{array}$ & $\begin{array}{l}\text { Mínimo } \\
\left(\mathrm{g} / \mathrm{cm}^{3}\right)\end{array}$ & W & GQ \\
\hline Downes et al. (1997) & $-1,225$ & 0,005 & 0,013 & $-0,026$ & $0,9799^{*}$ & $1,6538^{*}$ \\
\hline Convencional & 0,588 & 0,004 & 0,012 & $-0,009$ & $0,9877^{\star}$ & $1,2279^{*}$ \\
\hline 1 & 0,086 & 0,005 & 0,021 & $-0,014$ & $0,9929^{\text {ns }}$ & $1,5236^{\mathrm{ns}}$ \\
\hline 2 & 0,119 & 0,005 & 0,021 & $-0,014$ & $0,9938^{\text {ns }}$ & $1,5318^{*}$ \\
\hline 3 & 0,157 & 0,005 & 0,021 & $-0,014$ & $0,9943^{\text {ns }}$ & $1,5410^{*}$ \\
\hline 4 & 0,083 & 0,004 & 0,009 & $-0,011$ & $0,9894^{\mathrm{ns}}$ & $1,306^{\mathrm{ns}}$ \\
\hline 5 & 0,122 & 0,004 & 0,009 & $-0,011$ & $0,9664^{\text {ns }}$ & $1,5381^{\star}$ \\
\hline 6 & 0,155 & 0,004 & 0,010 & $-0,010$ & $0,9911^{\mathrm{ns}}$ & $1,3043^{\mathrm{ns}}$ \\
\hline 7 & 0,029 & 0,003 & 0,009 & $-0,011$ & $0,9891^{\text {ns }}$ & $1,3251^{\mathrm{ns}}$ \\
\hline 8 & 0,068 & 0,003 & 0,009 & $-0,011$ & $0,9900^{\text {ns }}$ & $1,3198^{\mathrm{ns}}$ \\
\hline 9 & 0,100 & 0,003 & 0,009 & $-0,010$ & $0,9908^{\mathrm{ns}}$ & $1,3219^{\text {ns }}$ \\
\hline 10 & 0,065 & 0,005 & 0,017 & $-0,014$ & $0,9955^{\mathrm{ns}}$ & $1,0876^{\mathrm{ns}}$ \\
\hline 11 & $-0,025$ & 0,003 & 0,008 & $-0,011$ & $0,9897^{\text {ns }}$ & $1,3153^{\text {ns }}$ \\
\hline 12 & 0,014 & 0,003 & 0,008 & $-0,010$ & $0,9902^{\text {ns }}$ & $1,3097^{\mathrm{ns}}$ \\
\hline 13 & 0,047 & 0,003 & 0,009 & $-0,010$ & $0,9914^{\mathrm{ns}}$ & $1,3118^{\mathrm{ns}}$ \\
\hline 14 & 0,011 & 0,005 & 0,016 & $-0,014$ & $0,9953^{\text {ns }}$ & $1,0741^{\mathrm{ns}}$ \\
\hline 15 & $-0,043$ & 0,005 & 0,016 & $-0,015$ & $0,9952^{\text {ns }}$ & $1,0772^{\mathrm{ns}}$ \\
\hline 16 & 0,078 & 0,005 & 0,011 & $-0,011$ & $0,9929^{\text {ns }}$ & $1,2057^{\mathrm{ns}}$ \\
\hline 17 & 0,117 & 0,005 & 0,011 & $-0,010$ & $0,9924^{\text {ns }}$ & $1,1899^{\text {ns }}$ \\
\hline 18 & 0,150 & 0,005 & 0,011 & $-0,010$ & $0,9925^{\text {ns }}$ & $1,1826^{\mathrm{ns}}$ \\
\hline 19 & 0,114 & 0,005 & 0,015 & $-0,014$ & $0,9984^{\text {ns }}$ & $0,9390^{\mathrm{ns}}$ \\
\hline 20 & 0,060 & 0,005 & 0,015 & $-0,014$ & $0,9982^{\text {ns }}$ & $0,9403^{\text {ns }}$ \\
\hline 21 & 0,006 & 0,005 & 0,014 & $-0,015$ & $0,9980^{\text {ns }}$ & $0,9328^{\mathrm{ns}}$ \\
\hline
\end{tabular}

$\mathrm{S}_{\mathrm{xy}}$ : desvio padrão; $\mathrm{W}^{*} \mathrm{e} \mathrm{GQ}^{*}$ : ausência de normalidade e homocedasticidade dos desvios, respectivamente.
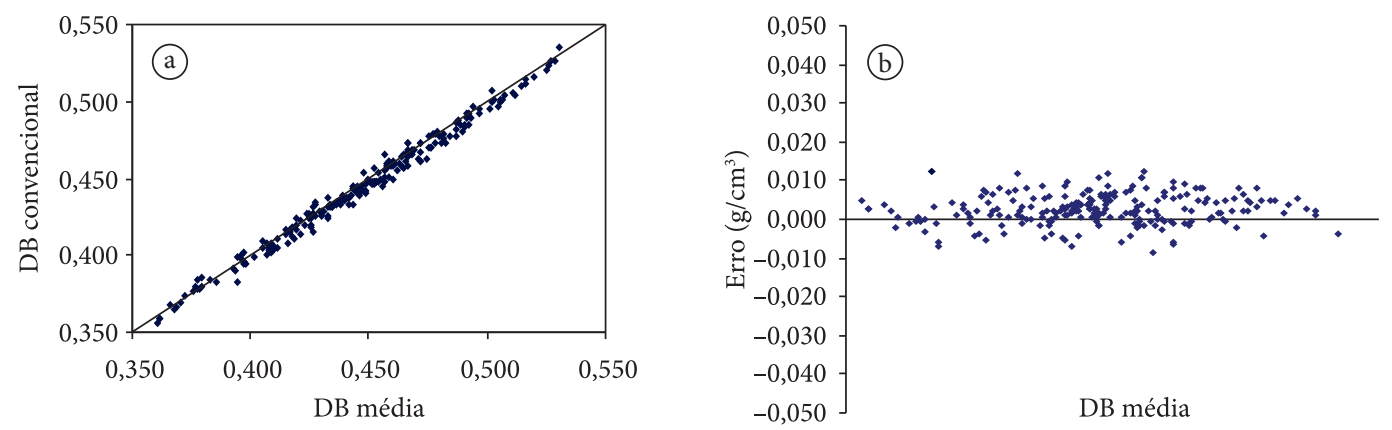

Figura 8. Relação entre os dados de DBmed e DB determinada pela amostragem convencional (a) e distribuição gráfica dos desvios (b).

Figure 8. Relation between data of the average $\mathrm{BD}$ and $\mathrm{BD}$ determined by conventional sampling (a) and graphic distribution of the deviations (b).

\section{CONCLUSÕES}

O modelo polinomial de $5^{\circ}$ ordem foi o que melhor representou o padrão de variação longitudinal da densidade básica, descrito pela seguinte tendência: decréscimo inicial, acréscimo e decréscimo final.

A densidade básica a $1,30 \mathrm{~m}$ do solo não foi a mais adequada para representar a densidade básica média do indivíduo em clones de Eucalyptus sp., 
sendo a mais adequada a altura relativa de $79 \%$ da altura comercial.

As alternativas amostrais de Downes et al. (1997) e a convencional podem não ser as mais adequadas para representar a densidade básica média do indivíduo. A amostragem convencional tendeu a subestimar a DBmed, enquanto que a amostragem proposta por Downes et al. (1997) tendeu a superestimar a DBmed.

As alternativas amostrais 7 (5, 25, 27, 78 e 79\%), $11(5,26,27,78$ e $79 \%)$ e $12(5,26,27,78$ e $80 \%)$ foram consideradas mais adequadas comparativamente à amostragem convencional e à proposta por Downes et al. (1997).

\section{AGRADECIMENTOS}

Os autores agradecem ao CNPq, FAPEMIG e Capes o apoio financeiro a esta pesquisa e à empresa ArcelorMittal Bioenergia Ltda. a doação do material biológico utilizado.

\section{STATUS DA SUBMISSÃO}

Recebido: 30/08/2011

Aceito: 21/02/2012

Resumo publicado online: 22/03/2012

Artigo completo publicado: 30/06/2012

\section{AUTOR(ES) PARA CORRESPONDÊNCIA}

\author{
Allan Motta Couto \\ Departamento de Ciências Florestais - DCF, \\ Universidade Federal de Lavras - UFLA, \\ Campus Universitário, CEP 37200-000, \\ Lavras, MG, Brasil \\ e-mail: allan_floresta@yahoo.com.br
}

\section{Thiago de Paula Protásio}

Departamento de Ciências Florestais - DCF, Universidade Federal de Lavras - UFLA, Campus Universitário, CEP 37200-000, Lavras, MG, Brasil e-mail: depaulaprotasio@gmail.com

\section{APOIO FINANCEIRO}

\section{REFERENNCIAS}

Arango Alzate SB, Tomazello Filho M, Piedade SMS. Variação longitudinal da densidade básica da madeira de clones de Eucalyptus grandis Hill ex Maiden, E. saligna Sm. e E. grandis x urophylla. IPEF 2005; (68): $87-$ 95.

Associação Brasileira de Normas Técnicas - ABNT. Normas. NBR-11941: Madeira - determinação da densidade básica. Rio de Janeiro: ABNT; 2003.

Couto AM, Neves TA, Protásio TP, Trugilho PF, Almado RP. Padrão de variação longitudinal da densidade básica em clones de Eucalyptus sp. In: Anais do XII Encontro Brasileiro em Madeiras e em Estruturas de Madeira; 2010; Lavras. Lavras: Universidade Federal de Lavras; 2010. CD-ROM.

Downes GM, Hudson IL, Raymond CA, Dean GH, Michell AJ, Schimleck R et al. Sampling plantation eucalypts for wood and fiber properties. Hobart: CSIRO; 1997.

Franco EJ, Scolforo JRS, Mello JM, Lima JT. Eficiência na estimativa do peso seco para árvores individuais e definição do ponto ótimo de amostragem para determinação da densidade básica de Eucalyptus camaldulensis. Ciência Florestal 1998; 8(1): 77-92.

Lima JT, Rosado SCS, Oliveira AD. Variação da densidade da madeira de Eucalyptus grandis, Eucalyptus terenticornis e Eucalyptus camaldulensis no sentido longitudinal dos caules. Ciência $e$ Prática 1992; 1(16): 123-127.

Mattos BD, Gatto DA, Stangerlin DM, Calegari L, Melo RR, Santini EJ. Variação axial da densidade básica da madeira de três espécies de gimnospermas. Revista Brasileira de Ciências Agrárias 2011; 6(1): 121-126.

Neves TA, Protásio TP, Couto AM, Trugilho PF, Silva VO, Vieira CMM. Avaliação de clones de Eucalyptus em diferentes locais visando à produção de carvão vegetal. Pesquisa Florestal Brasileira 2011; 31(68): 319:330.

Oliveira JTO, Hellmeister JC, Tomazello Filho M. Variação do teor de umidade e da densidade básica na madeira de sete espécies de eucalipto. Revista Árvore 2005; 29(1): 115-127. http://dx.doi.org/10.1590/ S0100-67622005000100013

Protásio TP, Couto AM, Reis AA, Neves TA, Trugilho PF. Análise da densidade básica da madeira de clones de Eucalyptus urophylla e Eucalyptus grandis. In: Anais do XII Encontro Brasileiro em Madeiras e em Estruturas de Madeira; 2010; Lavras. Lavras: Universidade Federal de Lavras; 2010. CD-ROM.

Protásio TP, Neves TA, Couto AM, Trugilho PF. Avaliação da densidade energética de clones de Eucalyptus em diferentes locais. In: Anais do VI Congresso Internacional de Bioenergia; 2011; Curitiba. 
Curitiba: Centro de Eventos FIEP/SESI; 2011. CDROM.

$\mathrm{R}$ Developement Core Team. R: A language and environment for statistical computing [online]. Vienna, Austria: R Foundation for Statistical Computing, 2009. [cited 2012 feb. 13] Available from: http://www.Rproject.org.

Trugilho PF, Arantes MDC, Pádua FA, Almado RP, Baliza AER. Estimativa de carbono fixado na madeira de um clone híbrido de Eucalyptus urophylla e Eucalyptus grandis. Cerne 2010; 16(Suplemento): 33-40. Trugilho PF, Paula R, Chaves MD, Cruz CR, Silva JRM, Pádua FA. Padrão de variação longitudinal da densidade básica da madeira em um clone híbrido de Eucalyptus. In: Anais do XI Encontro Brasileiro em Madeiras e em Estruturas de Madeira; 2008; Londrina. Londrina: Universidade Federal de Londrina; 2008. 